msh-mss Mathématiques et sciences humaines

144 | Hiver 1998

Varia

\title{
Inférence combinatoire en analyse géométrique des données
}

combinatorial inference in geometric data analysis

\section{Brigitte Le Roux}

\section{(2) OpenEdition}

12 Journals

Édition électronique

URL : http://journals.openedition.org/msh/2781

DOI : $10.4000 /$ msh.2781

ISSN : 1950-6821

Éditeur

Centre d'analyse et de mathématique sociales de l'EHESS

Édition imprimée

Date de publication : 1 décembre 1998

ISSN : 0987-6936

Référence électronique

Brigitte Le Roux, «Inférence combinatoire en analyse géométrique des données », Mathématiques et sciences humaines [En ligne], 144 | Hiver 1998, mis en ligne le 10 février 2006, consulté le 23 juillet 2020. URL : http://journals.openedition.org/msh/2781; DOI : https://doi.org/10.4000/msh.2781

Ce document a été généré automatiquement le 23 juillet 2020

(c) École des hautes études en sciences sociales 


\title{
Inférence combinatoire en analyse géométrique des données
}

Combinatorial inference in geometric data analysis

\author{
Brigitte Le Roux
}

\section{RÉSUMÉS}

Dans cet article, on se propose de montrer comment, en analyse géométrique des données (analyse des correspondances, analyse en composantes principales...) les statistiques descriptives utilisées comme aides à l'interprétation peuvent faire l'objet de procédures d'inférence combinatoire reposant sur des tests de permutation interprétés en termes de proportions d'échantillons plus extrêmes que les données, et qui prolongent directement la description statistique. Dans la première partie, on présente les tests de typicalité et d'homogénéité ; dans la deuxième partie, on les applique aux variables principales de l'analyse des correspondances multiples, en prenant pour population l'ensemble des individus.

In this paper, we aim at showing how, in Geometric Data Analysis (Correspondence Analysis, Principal Component Analysis...) descriptive statistics utilized as aids to interpretation can be used as combinatorial inference procedures based on permutation tests interpreted in terms of proportion of samples which are more extreme than the data. These procedures directly extend statistical description. In the first part, we will present typicality and homogeneity tests. In the second part, we will apply them to the principal variables provided by Multiple Correspondence Analysis, taking as the population the set of individuals. 
INDEX

Thèmes : combinatoire, données (analyse des), géométrie, inférence, statistique 